\title{
Ischemia miocardica e rilascio di ormoni vasoconstrittori nell'ipertensione associata ad insufficienza renale cronica: possibile ruolo della malattia coronarica dei piccoli vasi
}

\author{
C. Napoli ${ }^{1,2}$, F. Di Gregorio ${ }^{3,4}$, P. Sorice ${ }^{1}$, M. Leccese ${ }^{5}$, L. Mansi ${ }^{3}$, A. Liguori ${ }^{1}$
}

Dipartimento di Medicina, ${ }^{1}$ Divisione di Cardiologia-CCU, Divisione di Nefrologia ed Emodialisi, Ospedale Regionale dei Pellegrini, Napoli; ${ }^{2}$ Dipartimento di Medicina Clinica e Sperimentale, Università di Napoli Federico II; ${ }^{3}$ Istituto di Scienze Radiologiche, Servizio di Medicina Nucleare, II Università di Napoli; ${ }^{4}$ Divisione di Medicina Nucleare, Ospedale di Udine; ${ }^{5}$ Divisione di Medicina Interna, Policlinico Casilino, Roma

ipertensione arteriosa induce alterazioni sia sulla geometria cardiaca sia sul flusso coronarico causando un elevato rischio cardiovascolare (1). L'ipertrofia ventricolare sinistra (IVS) è responsabile di un aumento dell'incidenza di morbidità e mortalità per eventi cardiovascolari (2), e rappresenta un rischio indipendente nel paziente uremico dove spesso si presenta nella forma asimmetrica (3-5).

Il complesso scenario fisiopatologico che accompagna il paziente affetto da ipertensione arteriosa, comprende anche il rilascio di fattori ormonali. D'altra parte, anche l'insufficienza renale cronica (IRC) influenza di per sé la risposta neuroendocrina (6-8). Pertanto nei pazienti uremici ipertesi si osserva una condizione patologica e neuroendocrina derivante sia dalla ipertensione arteriosa sia dalla IRC. In tale contesto, diversi mediatori bioumorali partecipano alla patogenesi della ischemia miocardica (9). Inoltre, è stato suggerito come una iperattività sim- patica sia in correlazione con l'IVS in pazienti uremici (10). Alti livelli del peptide vasoconstrittore endotelina-1 (ET-1) sono stati riscontrati nell'ipertesione arteriosa (11-13) e nell'IRC $(13,14)$. Ma sia l'ET-1 (15-21) sia l'iperattività simpatica (22-24) sembrano essere implicati nell'ischemia miocardica, ed un ruolo diretto dell'adrenalina è stato ipotizzato nella formazione della placca aterosclerotica (25). Infine, l'ET-1 aumenta l'azione vasoconstrittrice della norepinefrina nell'uomo (18). Queste evidenze suggeriscono che 1'ET-1 ed il sistema simpatico possano giocare un ruolo nella patogenesi dell'ischemia miocardica in pazienti ipertesi affetti da IRC. In uno studio precedente, abbiamo dimostrato come questa ipotesi sia corretta (26). Lo scopo del presente studio è stato di ampliare la casistica studiata e di includere un elevato numero di pazienti con studio coronarografico al fine di indagare la causa dell'aumentata incidenza di ischemia miocardica in questi pazienti.

\section{Metodi}

\section{Pazienti}

Abbiamo selezionato 65 controlli affetti da ipertesione essenziale e 42 pazienti affetti da ipertensione arteriosa e concomitante IRC. Pazienti erano esclusi dallo studio se affetti da diabete, ipertensione secondaria, cardiomiopatie primitive, valvulopatie, aritmie atriali e ventricolari, o con età $>72$ anni. Al fine di evitare disparità in partenza tra $\mathrm{i}$ due gruppi, la cardiopatia ischemica era indagata dal rilievo della storia anamnestica, ECG a riposo e da sforzo, thallium-201 imaging, ed ecocardiografia standard. Le cause di IRC erano: glomerulonefrite cronica $(n=18)$, nefrite interstiziale $(n=12)$, rene policistico $(n=12) .36$ dei 42 pazienti ipertesi con IRC erano in trattamento con emodialisi in buffer acetato. I due gruppi di pazienti ipertesi avevano una pressione arteriosa $>150 / 95 \mathrm{~mm} \mathrm{Hg}$ rilevata a più riprese durante il controllo 
ambulatoriale, in assenza di trattamento farmacologico.

\section{Parametri ecocardiografici}

I pazienti ipertesi essenziali erano studiati di routine. I pazienti ipertesi con IRC erano esaminati 24 ore dopo la sessione dialitica. B-mode e M-mode Eco-Doppler erano eseguiti con sonde da 2.25 a $3.0 \mathrm{MHz}$ ed i tracings erano registrati a $50 \mathrm{~mm} / \mathrm{sec}$. Le misurazioni erano effettuate secondo le indicazioni fornite dall'American Society of Echocardiography (27), e precedentemente descritte in dettaglio $(5,26)$. La wall-motion era misurata con la suddivisione in 24 segmenti del miocardio (26). Per ogni segmento si faceva riferimento ad una 5-point scale $(0=$ normal, $1=$ mild hypokinetic, $2=$ moderate hypokinetic, $3=$ severe ipokinetic, 4= akinetic). Veniva anche effettuata la misurazione computerizzata del wall thickening (28) definita come (\%WT):

T end-systolic - T end-diastolic x 100

T end-diastolic

La procedura in dettaglio è stata precedentemente descritta dal nostro gruppo (26). Gli archi da $0^{\circ}$ a $180^{\circ}$ erano $1^{\prime}$ espressione di segmenti anteriori miocardici (settale, anteriore, laterale), mentre gli archi da $180^{\circ}$ a $360^{\circ}$ erano rappresentativi della porzione posteriore (28).

\section{Scintigrafia miocardica}

La ${ }^{99 m}$ Tc-methoxy-isobutyl-isonitril (MIBI) single photon emission computed tomography (SPECT) imaging è stata effettuata secondo il same-day protocol ed utilizzando la sequenza rest-stress (29). La precisa struttura del MIBI è ${ }^{99 \mathrm{~m}} \mathrm{Tc}$ (MIBI) $^{6+}$ (Cardiolite, Du Pont Radipharmaceutical, Billerica, MA, U.S.A.), dove il MIBI è 2-methoxy-isobutyl-isonitrile. A riposo $7 \mathrm{mCi}$ di ${ }^{99 \mathrm{~m}} \mathrm{Tc}-\mathrm{MIBI}$ erano iniettati endovena. La procedura di acquisizione delle immagini da parte di una camera rotante (Siemens Orbiter 75, Germany) è stata descritta in dettaglio dal nostro gruppo (26). L'uptake di ${ }^{99 \mathrm{~m} T c-}$ MIBI era valutato mediante computer sulla base di un visual score con un 5point grading system $(0=$ normal, $1=$ mild reduction, $2=$ moderate reduction, $3=$ severe reduction and $4=$ absent uptake). L'ischemia miocardica segmentale era definita mild ischemia $\left(\mathrm{MI}_{1}\right)$ (stress/rest segmental score: $2 / 0,3 / 0$, $4 / 1,4 / 0)$, moderate ischemia $\left(\mathrm{MI}_{2}\right)$ (stress/rest segmental score: 2/1, 3/1, $4 / 2$ ), severe ischemia (SI) (stress/rest segmental score: $3 / 2,4 / 3$ ) o scar (S) (stress/rest segmental score: $2 / 2,3 / 3$, 4/4). I pazienti erano sottoposti a test da sforzo su cicloergometro al fine di raggiungere un doppio prodotto di almeno 25000 o l'80\% della frequenza cardiaca.

\section{Ormoni}

La concentrazione nel plasma di norepinefrina e di endotelina-1 (ET-1) era valutata mediante RIA, come precedentemente descritto $(30,31)$. Il within-assay precision era dell' $11.6 \%$ ed il betweenassay precision era del $10.3 \%$. La sensitività dell' ET-1 assay era di 1.2 pg/mL.

\section{Design dello Studio}

Questo è un cross-sectional study. Tutti i pazienti interrompevano la terapia medica per almeno 5 giorni prima dello studio. Non vi erano differenze significative tra le terapie mediche impiegate nei due gruppi studiati. La funzionalità cardiaca era valutata ecocardiograficamente mediante due metodiche (wall motion and wall thickening analysis) eseguite a riposo ed all'acme dello sforzo. I medici nucleari eseguivano l'analisi MIBI-SPECT in cieco rispetto ai dati ecocardiografici. Le concentrazioni ormonali venivano determinate a riposo e all'acme dello sforzo. Lo studio era approvato dal Comitato Etico dell'Ospedale Regionale dei Pellegrini. I pazienti davano il consenso informato allo studio.

\section{Statistical analysis}

I dati erano analizzati in cieco rispetto alle metodiche impiegate (ecocardiografia e MIBI-SPECT) e rispetto ai due gruppi di pazienti. I dati erano espressi come media \pm standard deviation. Le comparazioni statistiche erano effettuate mediante Student t-test con Bonferroni's correction. Le correlazioni erano effettuate mediante least square analysis. Il Wilcoxon's one-sample rank sum test per dati appaiati era utilizzato per comparare i dati a riposo a quelli dello sforzo. L'agreement tra la MIBI-SPECT e l'ecocardiografia era valutata con la kappa statistic. Lo Spearman's test (rank correla- tion) era impiegato per valutare le modifiche dei valori di ET-1 rispetto ai MIBI defects scores. La significatività statistica era accettata al $95 \%$ del confidence interval $(\mathrm{p}<0.05)$.

\section{Risultati}

\section{Pazienti}

Il carico raggiunto durante il test da sforzo era simile nei due gruppi (Tab. I). Non vi erano differenze significative tra i due gruppi di pazienti per quanto attiene le principali caratteristiche (Tab. II). Di contro i pazienti ipertesi con IRC avevano un più severo grado di IVS (Tab. II), come atteso dalla letteratura $(3-5,26)$. Il cardiac index era leggermente aumentato negli IRC e tale risultato è parzialmente dovuto alla fistola arteriovenosa $(3-5,32)$, ed in parte all'anemia (34). Tuttavia, i nostri pazienti erano in trattamento con eritropoietina che aboliva lo stato di anemia (Tab. II). La creatinina correlava positivamente con la misura del septal thickness $(\mathrm{r}=0.58 ; \mathrm{p}<0.01)$.

\section{MIBI-SPECT and echocardiographic analysis}

L'analisi segmentale mostrava una significativa tendenza ad un maggiore grado di ischemia da sforzo nei pazienti ipertesi con IRC (Tab. III e Fig. 1). L'analisi globale dei risultati ha dimostrato che tutti i segmenti miocardici erano interessati dal fenomeno senza prevalenza di una regione particolare (Tab. III). Nella Tabella IV si mostra la correlazione dei dati sulla cinetica miocardica forniti dall'ecocardiografia con la simultanea analisi segmentale della perfusione alla SPECT. Tali risultati ottenevano un'alta significatività statistica (Kappa statistics $=0.68, \mathrm{p}<0.001)$. La misurazione del wall thickening, come menzionato, è indipendente dagli assi di riferimento e dall'abilità dell'operatore (28). Le regioni miocardiche con un systolic thickening $<80 \%$ rispetto al baseline venivano considerate come ischemiche. La Figura 2 mostra come il \% di wall thickening era significativamente più basso nei pazienti ipertesi con IRC. Tali risultati confermano ulteriormente come in questi pazienti il grado di ischemia miocardica cinetica risulti maggiore. Durante lo sforzo, il wall thickening correlava anche con il 
wall motion score $(r=0.73, p<0.01)$ ed il MIBI ischemic grade $(\mathrm{r}=0.80, \mathrm{p}<0.001)$.

\section{Anatomia coronarica}

Al fine di identificare la causa dell'ischemia miocardica, alla fine dello studio 19 pazienti ipertesi IRC e 40 pazienti con IE con segni di moderata e severa ischemia erano sottoposti ad esame coronarografico (Tab. V). I risultati indicavano un significativo basso indice di lesioni coronariche emodinamicamente significative nei pazienti ipertesi con IRC rispetto ai pazienti con sola IE.

\section{Ormoni vasoconstrittori}

La Tabella VI mostra i risultati relativi al rilascio ormonale determinato a riposo ed in seguito a sforzo al cicloergometro. $\mathrm{La}$ concentrazione basale plasmatica di norepinefrina era significativamente elevata nei pazienti IRC e si manteneva tale dopo lo sforzo. Simili dati erano ottenuti con le concentrazioni plasmatiche di ET-1.

Dopo lo sforzo vi era una significativa correlazione tra l'incremento nella concentrazione della norepinefrina $(r=0.55$, $\mathrm{p}<0.01$ ) ed i segmenti ischemici alla MIBI-SPECT nei pazienti ipertesi con IRC. L'incremento nella concentrazione di ET-1 e SPECT era positivo nei due gruppi (IE: $r=0.45, p<0.05$; IRC: $r=0.63$, $\mathrm{p}<0.01)$. Infine, la condizione di IRC (creatinina) correlava con i livelli di ET$1(\mathrm{r}=0.37 ; \mathrm{p}<0.05)$.

\section{Discussione}

Il nostro studio conferma il dato che $i$ pazienti ipertesi con IRC hanno un'elevata incidenza di ischemia miocardica (26) valutata con MIBI-SPECT e funzionalmente mediante lo studio ecocardiografico della contrattilità miocardica (wallmotion e wall thickening). Lo studio simultaneo di questi due indici altamente correlati (33) risulta anche associato a ridotto consumo di acidi grassi $(34,35)$. L'aumento della casistica ha anche confermato che l'ischemia miocardica era anche associata ad aumento significativo di norepinefrina ed ET-1 (26), due importanti ormoni vasoconstrittori. In questo studio abbiamo anche eseguito esame coronarografico nei pazienti che avevano presentato segni di ischemia miocardica. I risultati hanno dimostrato come il
TAB. I - PARAMETRI EMODINAMICI DURANTE IL TEST DA SFORZO NEI DUE GRUPPI DI PAZIENTI

\begin{tabular}{lccccc}
\hline & \multicolumn{2}{c}{ IE } & \multicolumn{2}{c}{ IRC } & \\
\hline & Riposo & Stress & Riposo & Stress & p \\
\hline Maximal Workload (W) & & 100 & & 100 & NS \\
& & $(75-125)$ & & $(75-125)$ & \\
Pressione sistolica (mmHg) & $171 \pm 18$ & $232 \pm 19$ & $173 \pm 19$ & $227 \pm 20$ & NS \\
Frequenza (b/min) & $77 \pm 8$ & $142 \pm 28$ & $75 \pm 9$ & $149 \pm 29$ & NS \\
\hline
\end{tabular}

TAB. II - CARATTERISTICHE DEI PAZIENTI

\begin{tabular}{|c|c|c|c|}
\hline & IRC & IE & p Value \\
\hline n & 42 & 65 & \\
\hline Età (anni) & $54 \pm 11$ & $57 \pm 10$ & ns \\
\hline Sesso (M:F) & $28: 14$ & $41: 24$ & \\
\hline Body Surface $\left(\mathrm{m}^{2}\right)$ & $1.9 \pm 0.2$ & $1.8 \pm 0.2$ & ns \\
\hline Durata dell'ipertensione (anni) & $7.9 \pm 2.6$ & $8.5 \pm 3.5$ & ns \\
\hline Creatinina $(\mathrm{mg} / \mathrm{dl})$ & $6.5 \pm 1.5$ & $1.0 \pm 0.3$ & $\mathrm{p}<0.003$ \\
\hline Clearance Creatinina ( $\mathrm{ml} / \mathrm{min})$ & $16.5 \pm 9.2$ & $109 \pm 19$ & $\mathrm{p}<0.001$ \\
\hline Hemoglobin $(\mathrm{g} / \mathrm{dl})$ & $12.2 \pm 0.9$ & $12.6 \pm 0.8$ & ns \\
\hline Hematocrit $(\%)$ & $37 \pm 3$ & $43 \pm 4$ & $\mathrm{p}<0.05$ \\
\hline Total cholesterol (mg/dl) & $250 \pm 28$ & $219 \pm 24$ & $\mathrm{p}<0.05$ \\
\hline LDL-cholesterol (mg/dl) & $149 \pm 25$ & $115 \pm 18$ & $\mathrm{p}<0.05$ \\
\hline HDL-cholesterol (mg/dl) & $34 \pm 4$ & $35 \pm 3$ & ns \\
\hline Tryglicerides (mg/dl) & $231 \pm 29$ & $181 \pm 30$ & $\mathrm{p}<0.05$ \\
\hline Systolic pressure (mmHg) & $171 \pm 18$ & $173 \pm 19$ & ns \\
\hline Diastolic pressure $(\mathrm{mmHg})$ & $120 \pm 9$ & $119 \pm 10$ & ns \\
\hline Heart rate (beats/min) & $77 \pm 8$ & $75 \pm 9$ & ns \\
\hline Cardiac index $\left(\mathrm{L} / \mathrm{min} / \mathrm{m}^{2}\right)$ & $3.2 \pm 0.6$ & $3.0 \pm 0.5$ & ns \\
\hline Stroke index $\left(\mathrm{ml} / \mathrm{min} / \mathrm{m}^{2}\right)$ & $38 \pm 8$ & $37 \pm 9$ & ns \\
\hline Total peripheral resistance $\left(\mathrm{U} / \mathrm{m}^{2}\right)$ & $45 \pm 15$ & $46 \pm 16$ & ns \\
\hline $\mathrm{LV}$ stroke work $\left(\mathrm{mmHg} \mathrm{mL} / \mathrm{m}^{2}\right)$ & $85 \pm 21$ & $84 \pm 19$ & ns \\
\hline Fractional fiber shortening (\%) & $37 \pm 6$ & $43 \pm 7$ & $\mathrm{p}<0.05$ \\
\hline Wall thickness/radius ratio & $0.7 \pm 0.05$ & $0.5 \pm 0.03$ & $\mathrm{p}<0.05$ \\
\hline LV muscle mass index ( $\mathrm{g} / \mathrm{BSA})$ & $180 \pm 25$ & $134 \pm 20$ & $\mathrm{p}<0.01$ \\
\hline Septal thickness (mm) & $15.2 \pm 2.3$ & $11.2 \pm 2.1$ & $\mathrm{p}<0.01$ \\
\hline Posterior wall thickness (mm) & $11.0 \pm 1.8$ & $10.8 \pm 1.9$ & ns \\
\hline LV end-diastolic diameter $\left(\mathrm{cm} / \mathrm{m}^{2}\right)$ & $2.4 \pm 0.1$ & $2.3 \pm 0.2$ & ns \\
\hline LV ejection fraction $(\%)$ & $56 \pm 4$ & $55 \pm 5$ & ns \\
\hline End diastolic volume index $\left(\mathrm{ml} / \mathrm{m}^{2}\right)$ & $55 \pm 8$ & $58 \pm 7$ & ns \\
\hline $\begin{array}{l}\text { Velocity of circumferential fiber } \\
\text { shortening (circ/sec) }\end{array}$ & $1.30 \pm 0.2$ & $1.44 \pm 0.3$ & $\mathrm{p}<0.05$ \\
\hline
\end{tabular}


TAB. III - ANALISI SEGMENTALE DI 100 SEGMENTI DI 42 PAZIENTI IRC E 65 PAZIENTI IE

\begin{tabular}{|c|c|c|c|c|c|}
\hline & Anteriore & Laterale & Inferiore & Setto & Apice \\
\hline \multicolumn{6}{|c|}{ IRC } \\
\hline $\mathrm{N}$ & 9 & 11 & 8 & 10 & 10 \\
\hline $\mathrm{MI}_{1}$ & $6^{*}$ & 5 & 5 & $7 *$ & 6 \\
\hline $\mathrm{MI}_{2}$ & 2 & $4 *$ & $6 *$ & 3 & $3 *$ \\
\hline $\mathrm{SI}^{2}$ & $2 *$ & 0 & 1 & 0 & 1 \\
\hline S & 1 & 0 & 0 & 0 & 0 \\
\hline \multicolumn{6}{|c|}{ IE } \\
\hline $\mathrm{N}$ & 14 & 14 & 12 & 15 & 15 \\
\hline $\mathrm{MI}_{1}$ & 3 & 4 & 5 & 3 & 4 \\
\hline $\mathrm{MI}_{2}$ & 2 & 2 & 2 & 2 & 1 \\
\hline SI & 0 & 0 & 1 & 0 & 0 \\
\hline S & 1 & 0 & 0 & 0 & 0 \\
\hline
\end{tabular}

Legenda: $N$ : normal tracer uptake; $\mathrm{MI}_{1}$ : mild ischemia; $\mathrm{MI}_{2}$ : moderate ischemia; SI: severe ischemia; $S$ : scar. ${ }^{*}=p<0.05$ vs IE

\section{TAB. IV - ANALISI COMPARATIVA SEGMENTALE TRA ECOCARDIOGRA- FIA (WALL MOTION SCORE) E SPECT ALL'ACME DELLO SFOR- ZO NEI DUE GRUPPI DI PAZIENTI}

\section{ECOCARDIOGRAFIA}

\begin{tabular}{lrrrrl}
\hline SPECT & 0 & 1 & 2 & 3 & 4 \\
\hline \multicolumn{7}{c}{ IRC (n= $\mathbf{4 2})$} \\
\hline 0 & 27 & 3 & 0 & 0 & 0 \\
1 & 0 & 36 & 1 & 0 & 0 \\
2 & 0 & 0 & 25 & 3 & 0 \\
3 & 0 & 0 & 2 & 3 & 0 \\
4 & 0 & 0 & 0 & 0 & 1 \\
\hline
\end{tabular}

IE (n=65)

\begin{tabular}{rrrrll}
\hline 0 & 56 & 1 & 2 & 0 & 0 \\
1 & 0 & 19 & 1 & 0 & 0 \\
2 & 0 & 0 & 15 & 1 & 0 \\
3 & 0 & 0 & 0 & 4 & 0 \\
4 & 0 & 0 & 0 & 0 & 1 \\
\hline
\end{tabular}

Exact agreement $91 \%$, kappa $=0.68, p<0.001$

78.9\% dei pazienti ipertesi con IRC non presentasse lesioni coronariche emodinamicamente significative. Questo dato suggerisce che la causa dell'ischemia miocardica in questi pazienti possa essere dovuta alla malattia coronarica dei piccoli vasi (small vessel intramyocardial disease). Questa condizione fisiopatologica contribuisce all'aumento del ri- schio cardiovascolare nei pazienti ipertesi con associata IRC.

I pazienti ipertesi con IRC sono soggetti a diversi disturbi cardiovascolari, ad esempio una precoce disfunzione diastolica (36), che risulta associata ad elevati livelli di ET-1 (37). D'altra parte, nell'ipertensione arteriosa la riserva coronarica è ridotta con la presenza della IVS
(38-40). L'IVS può quindi determinare ulteriori richieste di ossigeno, in special modo se coesiste la malattia coronarica dei piccoli vasi (41). Poiché i nostri pazienti ipertesi con IRC avevano una significativa IVS, questa condizione può predisporli ad ulteriori richieste metaboliche che complicherebbero la small vessel intramyocardial disease.

L'indagine ventricolografica radionuclidica può presentare alcune difficoltà a causa della presenza di IVS (42). Pertanto, la MIBI-SPECT è l'indagine di prima scelta in questa situazione (43). La differenza di IVS tra i due gruppi poteva deporre a favore di una differente riserva coronarica come possibile causa dell'ischemia miocardica. Tuttavia, tale ischemia risultava distribuita omogeneamente nel miocardio. Inoltre, l'esame coronarografico ha scartato questa possibilità. I nostri dati sono indirettamente supportati dall'evidenza che l'angina pectoris nei pazienti uremici ha un quadro coronarografico normale (44), e che in un modello sperimentale di uremia si assiste ad una decremento della vascolarizzazione (45). Infine, i dati dei pazienti ipertesi con IRC sono molto simili per incidenza di ischemia miocardica a quelli dei pazienti ipertesi con aldosteronismo primario (46) suggerendo una possibile spiegazione patogenetica comune.

In accordo con la letteratura (47) i nostri pazienti con IRC presentavano una moderata dislipidemia, che poteva contribuire alla maggiore incidenza di ischemia miocardica. Sia la norepinefrina sia l'ET-1 sono state associate allo sviluppo della cardiopatia ischemica $(9,15,17$, 18, 20-23). Alti livelli di ET-1 sono anche associati all'ipertensione arteriosa ed all'uremia cronica $(14,15)$. Questi ormoni vasoconstrittori possono interagire tra di loro e favorire l'ischemia miocardica. A tal riguardo, l'infusione centrale (48) e periferica (17) di ET-1 potenzia l'azione della norepinefrina, mentre l'ET-1 produce anche effetti diretti sul circolo coronarico (48). Pertanto, l'abnorme rilascio di ET-1 e di norepinefrina, sia in condizione di riposo sia dopo lo sforzo, può contribuire a generare ischemia miocardica in pazienti ipertesi con IRC.

In conclusione, $\mathrm{i}$ nostri risultati confermano che i pazienti ipertesi con IRC hanno un'elevata incidenza di ischemia miocardica associata ad aumento significativo del rilascio di norepinefrina ed ET-1. Lo studio coronarografico ha mo- 
TAB. V - ANATOMIA CORONARICA DEI PAZIENTI

\begin{tabular}{|c|c|c|c|}
\hline & IRC $(n=19)$ & IP $(n=40)$ & $\mathbf{p}$ \\
\hline Assenza di lesioni, n (\%) & $9(47.36)$ & $10(25.0)$ & $<0.05$ \\
\hline $\begin{array}{l}\text { Presenza di placche non } \\
\text { emodinamicamente significative } \\
\text { ( }<50 \text { del lume vasale), } \mathrm{n}(\%)\end{array}$ & $6(31.57)$ & $8(20.0)$ & $\mathrm{ns}$ \\
\hline $\begin{array}{l}\text { Numero totale di pazienti senza } \\
\text { lesioni significative, } \mathrm{n}(\%)\end{array}$ & $15(78.95)$ & $18(45.0)$ & $<0.05$ \\
\hline $\begin{array}{l}\text { Numero totale di pazienti con } \\
\text { lesioni significative, } \mathrm{n}(\%)\end{array}$ & $4(21.05)$ & $22(55.0)$ & $<0.001$ \\
\hline Discendente anteriore, $\mathrm{n}(\%)$ & $2(9.1)$ & $16(40.0)$ & $<0.001$ \\
\hline Circonflesso, n (\%) & $2(9.1)$ & $11(27.5)$ & $<0.001$ \\
\hline Coronaria destra, $\mathrm{n}(\%)$ & $2(9.1)$ & $6(15.0)$ & $<0.05$ \\
\hline
\end{tabular}

TAB. VI - RILASCIO ORMONALE DURANTE RIPOSO E STRESS NEI DUE GRUPPI DI PAZIENTI

\begin{tabular}{lcccc}
\hline & \multicolumn{2}{c}{ RIPOSO } & \multicolumn{2}{c}{ STRESS } \\
\hline & IE & IRC & IE & IRC \\
\hline Norepinefrina $(\mathrm{pg} / \mathrm{ml})$ & $285 \pm 115$ & $418 \pm 165^{*}$ & $588 \pm 205 \dagger$ & $807 \pm 210^{*} \dagger$ \\
Endotelina- $1(\mathrm{pmol} / \mathrm{L})$ & $1.60 \pm 0.10$ & $1.75 \pm 0.11^{*}$ & $1.72 \pm 0.10$ & $1.94 \pm 0.12^{*} \dagger$ \\
\hline$*=p<0.05$ vs IE; $\dagger=p<0.05$ vs Riposo & & &
\end{tabular}

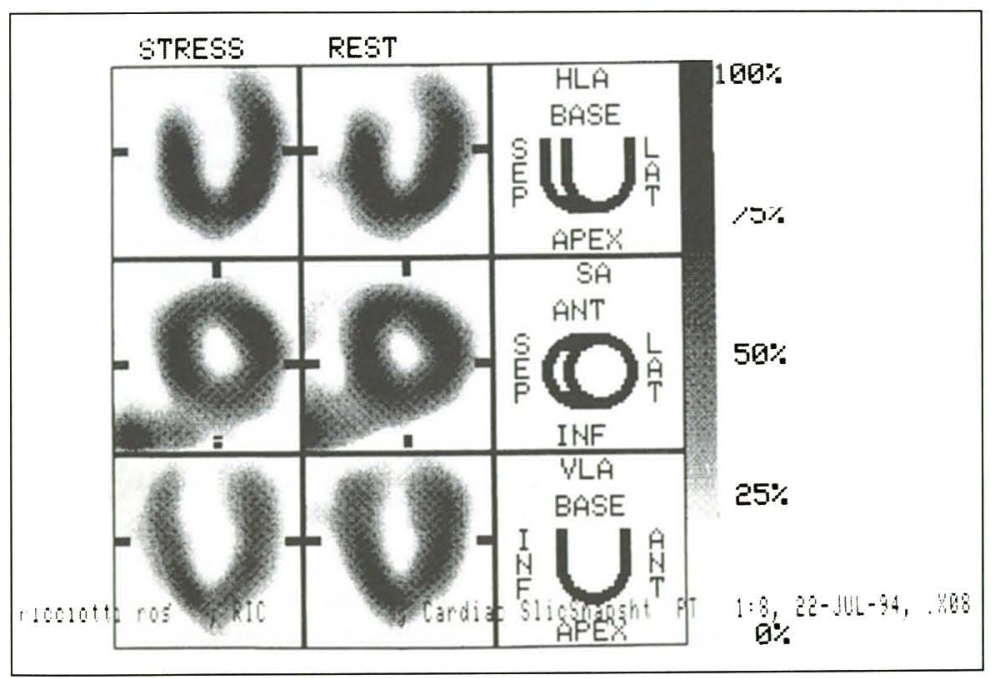

Fig. 1 - Esempio tipico di MIBI-SPECT in paziente iperteso con IRC. Ischemia da sforzo settale ed anterolaterale.

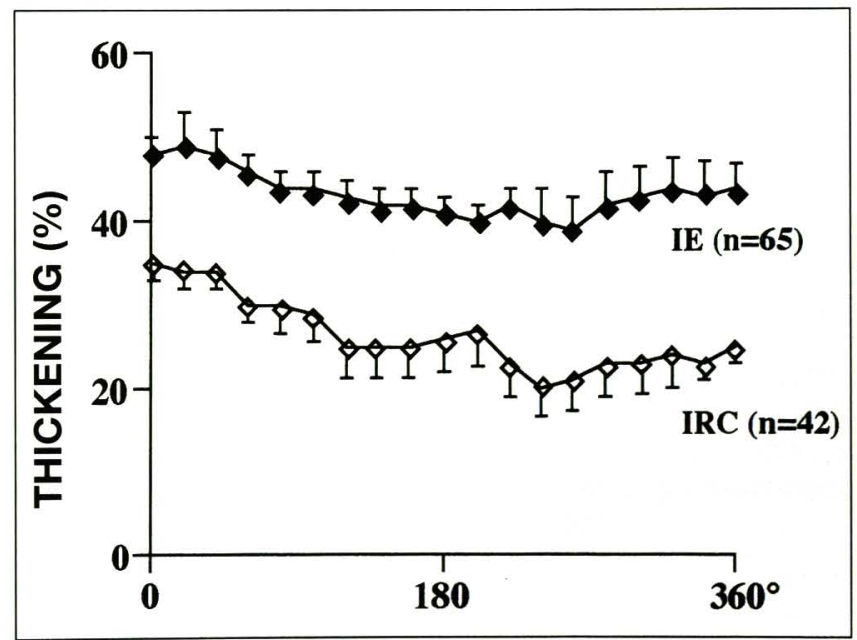

Fig. 2 - Percento di wall thickening in pazienti con ipertensione essenziale (IE) ed in pazienti ipertesi con insufficienza renale cronica (IRC). Dati ottenuti ecocardiograficamente all'acme dello sforzo. 
strato come il $78.9 \%$ dei pazienti ipertesi con IRC non presentava lesioni coronariche emodinamicamente significative suggerendo che la causa dell'ischemia miocardica in questi pazienti possa essere la malattia coronarica dei piccoli vasi (small vessel intramyocardial disease). Questa condizione fisiopatologica contribuisce all'aumento del rischio cardiovascolare in tali pazienti.

\section{BIBLIOGRAFIA}

1. Stamler J, Wentworth DN, Neaton JD. Blood pressure (systolic and diastolic) and risk of fatal coronary heart disease. Hypertension 1989; 13 (Suppl.1): 2S-12S.

2. Casale PN, Devereux RB, Milner $\mathrm{M}$, et al. Value of echocardiographic left ventricular mass in predicting cardiovascular morbid events in hypertensive men. Ann Intern Med 1986; 105: 173-8.

3. Huting J, Kramer W, Charra B, Laurent G, Wizemann V, Schutterle G. Asymmetric septal hypertrophy and left atrial dilatation in patients with end-stage renal disease longterm hemodialysis. Clin Nephrol 1989; 32: 276-83.

4. Silberberg JS, Barre PE, Prichard SS, Sniderman AD. Impact of left ventricular hypertrophy on survival in end-stage renal disease. Kidney Int 1989; 36: 286-90.

5. Paracuollo D, Esposito E, Di Benedetto A et al. Cardiac morphology evaluation in patients with chronic renal failure. In: Andreucci VE, Dal Canton A (Eds). New Therapeutic Strategies in Nephrology. Kluwer Academic Publishers, Norwell, MA, U.S.A., 1991: 162-8.

6. Cooke RC, Gann DS, Whelton $\mathrm{PK}$, et al. Hormonal responses to acute volume changes in anephric subjects. Kidney Int 1983; 23: 71-8.

7. Burges ED. Cardiac vagal denervation in hemodialysis patients. Nephron 1982; 30: 228-30.

8. Corder GN, Sharma J, McDonald RH. Variable levels of plasma catecholamines and dopamine betahydroxylase in hemodialysis patients. Nephron 1980; 25: 267-72.

9. Remme WJ, Kruyssen DA, Look MP, Bootsma M, de Leeuw PW. Systemic and cardiac neuroendocrine activation and severity of myocardial ischemia in humans. $\mathbf{J}$ Am Coll Cardiol 1994; 23: 82-91.
10. Bernardi D, Bernini L, Cini G, Ghione S, Bonechi I. Asymmetric septal hypertrophy and sympathetic overactivity in normotensive hemodialyzed patients. Am Heart J 1985; 109: 539-45.

11. Vanhoutte PM. Is endothelin involved in the pathogenesis of hypertension? Hypertension 1993; 21: 747-51.

12. Kohno M, Yasunari K, Murakawa KI, et al. Plasma immunoreactive endothelin in essential hypertension. Am J Med 1990; 88: 614-8.

13. Shichiri M, Hirata Y, Ando K, et al. Plasma endothelin levels in hypertension and chronic renal failure. Hypertension 1990; 15: 493-6.

14. Koyama H, Tabata T, Nishizawa Y, Inoue T, Morii H, Yamaj T. Plasma endothelin levels in patients with uremia. Lancet 1989; i: 991-2.

15. Yasuda M, Konho M, Thara A, et al. Circulating immunoreactive endothelin in ischemic heart disease. Am Heart J 1990; 119: 801-6.

16. Watanabe T, Suzuki N, Shimàmoto N, et al. Endothelin in myocardial infarction. Nature 1990; 334: 114.

17. Yang Z, Richard V, Von Segesser L, et al. Thresold concentrations of endothelin-1 potentiate contractions to norepinephrine and serotonin in human arteries: A new mechanism of vasospasm? Circulation 1990; 82: 188-95.

18. Toyo-oka T, Aizawa T, Suzuki $\mathrm{N}$, et al. Increased plasma level of endothelin-1 and coronary spasm induction in patients with vasospastic angina pectoris. Circulation 1991; 83: 476-83.

19. Clozel JP, Sprecher U. Influence of low perfusion pressure on effect of endothelin on coronary vasculary bed. Am J Physiol 1991; 260: H893-901.

20. Ray SG, McMurray JJ, Morton JJ, Dargie HJ. Circulating endothelin in acute ischemic syndromes. $\mathrm{Br}$ Heart J 1992; 67: 383-6.

21. Petersen CL, Hansen A, Frand- 
sen E, et al. Endothelin release and enhanced regional myocardial ischemia induced by cold-air inhalation in patients with stable angina. Am Heart J 1994; 128: 511-6.

22. Pepine CJ. Circadian variations in myocardial ischemia: implications for managment. JAMA 1991; 265: 386-90.

23. Rocco MB, Nabel EG, Selwyn AP. Circadian rhythms and coronary artery disease. Am J Cardiol 1987; 59: 13C-7C.

24. Amadi A, Ponikowski P, Coats AJS. Role of catecholamines and sympathetic activation as a risk factor for coronary artery disease. J Cardiovasc Risk 1995; 2: 222-8.

25. Helin P, Lorenzen I, Garbarsch $C$, Mathiesen ME. Arteriosclerosis in rabbit aorta induced by noradrenaline. Atherosclerosis 1970; 12: 125-32.

26. Napoli C, Di Gregorio F, Sorice $P$, et al. High prevalence of myocardial ischemia and vasoconstrictive hormonal release in hypertension during chronic renal failure. Nephron 1997; 76: 434-44.

27. Sahn DJ, DeMaria A, Kisslo J, Weyman A. The Committee on Mmode standardization of the American Society of Echocardiography. Recomendations regarding quantitation in M-mode echocardiography: Result of a survey of echocardiographic measuraments. Circulation 1978; 58: 1072-83.

28. Henschke CI, Risser TA, Sandor T, Hanlon WB, Neumann A, Wynne J. Quantitative computer-assisted analysis of left ventricular wall thickening and motion by 2 -dimensional echocardiography in acute myocardial infarction. Am J Cardiol 1983; 52: 960-4.

29. Leppo JA, DePuey EG, Johnson LL. A review of cardiac imaging with Sestamibi and Teboroxime. J Nucl Med 1991; 32: 2012-22.

30. Liguori A, Di Gregorio F, Napoli $\mathrm{C}$, et al. Atrial natriuretic factor and sympathetic activation in human heart failure. Eur J Pharmacol Sci 1994; 16:61-7.
31. Ciafrè SA, D'Armiento FP, Liguori A, et al. Angiotensin II evokes the release of ET-1 from human endothelial cells in vitro. Am J Clin Pathol 1992; 98: 379-80.

32. Liguori A, Di Gregorio F, Napoli C, et al. Effects of blood loading on left ventricular function in chronic uremics with arterio-venous fistula. Chest 1993; 103 (Suppl. A); 192S.

33. Smart SC. The clinical utility of echocardiography in the assessment of myocardial viability. J Nucl Med 1994; 35 (Suppl.): 49S-58S.

34. Franken PR, De Geeter F, Dendale P, Block P, Bossuyt A. Regional distribution of I-123 (otho-iodophenyl)-pentadecanoic acid and Tc${ }^{99 \mathrm{~m}}$ MIBI in relation to wall motion after thrombolysis for acute myocardial infarction. Nucl Med Commun 1993; 14: $310-7$

35. Tamaki N, Kawamoto M, Yonekura $\mathrm{Y}$, et al. Regional metabolic abnormality in relation to perfusion and wall motion in patients with myocardial infarction: assessment with emission tomography using an iodinated branched fatty acid analog. J Nucl Med 1992; 33: 659-67.

36. Liguori A, Napoli C, Sorice P, et al. Early alteration of left ventricular diastolic function in hypertensive patients on maintenance hemodialysis. Kidney Int 1993; 43: 759-60.

37. Napoli C, Liguori A, Sorice P, et al. Relations between vasoactive hormones and diastolic function in hypertensive uremics. J Intern Med 1996; 240: 1-6.

38. Foley RN, Parfrey PS, Harnett JD, Kent JM. Impact of hypertension on cardiomyopathy, morbidity, and mortality in end-stage renal disease. Kidney Int 1996; 49: 1379-83.

39. Houghton JF, Frank MJ, Carr AA, von Dohlen TW, Pisant LLM. Relations among impaired coronary flow reserve, left ventricular hypertrophy and thallium perfusion defects in hypertensive patients without obstructive coronary artery disease. J Am Coll Cardiol 1990; 15: 43-51.

40. Opherk D, Mall G, Zebe H, et al. Reduction of coronary reserve; a mechanism for angina pectoris in patients with arterial hypertension and normal coronary arteries. Circulation 1984; 69: 1-7.

41. James TN. Morphologic characteristics and functional significance of focal fibromuscular dysplasia of small coronary arteries. Am J Cardiol 1990; 65: $12 \mathrm{G}-22 \mathrm{G}$.

42. Wasserman AG, Katz RG, Varghese PJ, et al. Exercise radionuclide ventriculographic responses in hypertensive patients with chest pain. N Engl J Med 1984; 311:1276-80.

43. Guideline for clinical use of cardiac radionuclide imaging. Report of the American College of Cardiology/American Heart Association Task Force on assessment of diagnostic and therapeutic cardiovascular procedures. J Am Coll Cardiol 1995; 25: 521-47.

44. Rostand SG, Rutsky EA. Ischemic heart disease in chronic renal failure: Management considerations. Semin Dial 1989; 2: 98-101.

45. Amann K, Wiest G, Zimmer G, Gretz N, Ritz E, Mall G. Reduced capillary density in the myocardium of uremic rats. Kidney Int 1992; 42: 1079-85.

46. Liguori A, Sorice P, Di Gregorio F, Leccese M, Mansi L, Napoli C. Evidence of exercise-induced myocardial ischemia in patients with primary aldosteronism. Eur Heart $\mathbf{J}$ 1997; 18 (Suppl. A): 532S.

47. Wheeler DC, Sweny P, Varghese Z. Hyperlipidemia and atherosclerosis in chronic dialysis patients. In: Maher JF (Ed). Replacement of renal function by dialysis. Boston, Kluwer Academic Publishers, 1989: 798-807.

48. Falatico R, Underwood DC, Cheung WM, et al. Coronary vasoconstrictor and aritmogenic properties of endothelin in the dog. Pharmacologist 1989; 31: 150-5. 